\title{
A Distance Model of Intuitionistic Fuzzy Cross Entropy to Solve Preference Problem on Alternatives
}

\author{
Mei Li ${ }^{1,2}$ and Chong Wu ${ }^{1}$ \\ ${ }^{1}$ School of Management, Harbin Institute of Technology, Harbin 150001, China \\ ${ }^{2}$ School of Logistics Management and Engineering, Guangxi Teachers Education University, Nanning 530001, China
}

Correspondence should be addressed to Mei Li; shmilylm@sina.com

Received 3 October 2015; Accepted 10 January 2016

Academic Editor: Anna M. Gil-Lafuente

Copyright (C) 2016 M. Li and C. Wu. This is an open access article distributed under the Creative Commons Attribution License, which permits unrestricted use, distribution, and reproduction in any medium, provided the original work is properly cited.

In the field of decision-making, for the multiple attribute decision-making problem with the partially unknown attribute weights, the evaluation information in the form of the intuitionistic fuzzy numbers, and the preference on alternatives, this paper proposes a comprehensive decision model based on the intuitionistic fuzzy cross entropy distance and the grey correlation analysis. The creative model can make up the deficiency that the traditional intuitionistic fuzzy distance measure is easy to cause the confusion of information and can improve the accuracy of distance measure; meanwhile, the grey correlation analysis method, suitable for the small sample and the poor information decision-making, is applied in the evaluation. This paper constructs a mathematical optimization model of maximizing the synthesis grey correlation coefficient between decision-making evaluation values and decision-makers' subjective preference values, calculates the attribute weights with the known partial weight information, and then sorts the alternatives by the grey correlation coefficient values. Taking venture capital firm as an example, through the calculation and the variable disturbance, we can see that the methodology used in this paper has good stability and rationality. This research makes the decision-making process more scientific and further improves the theory of intuitionistic fuzzy multiple attribute decision-making.

\section{Introduction}

Professor Zadeh [1] pioneered the concept of fuzzy sets and opened up a new area for people to deal with the fuzzy information in 1965. On this basis Professor Atanassov [2, 3 ] put forward the concepts of intuitionistic fuzzy sets and interval valued intuitionistic fuzzy sets. In the traditional fuzzy set, only the membership degree is considered, and the intuitionistic fuzzy set not only considers the membership degree, but also considers the nonmembership degree and the uncertainty degree. As a result, it is more flexible than the fuzzy set in dealing with the uncertain problems and the fuzzy information. The content of the decision theory with the intuitionistic fuzzy information mainly contains the similarity and the distance measure $[4,5]$, the determination of the attribute weight [6-8], the application of the intuitionistic fuzzy multiple attribute method [9-12], and the calculation of the score function $[13,14]$. However, the intuitionistic fuzzy distance formulas used in these methods still lack sufficient comparison information which often makes the difference of the results very small so that it is difficult to make decisions.

Besides, in the practice of decision-making, to get more comprehensive and accurate understanding of the alternatives and to achieve the utility maximization better, decisionmakers often try to obtain the preference information of the alternatives by all means. The preference information on alternatives usually refers to the tendency and the emotion hidden inside the decision-makers. Rational decision-makers select alternatives in accordance with a predetermined optimization principle. Although their preference information contains subjective elements, the decision criterion is still based on the maximization of the expected utility. Therefore, the decision-making problem with preference information on alternatives still belongs to the multiple attribute decisionmaking problem under the complete rational perspective.

In recent years, scholars have studied multiple attribute decision-making problems of different data types with preference on alternatives, and such problems have become 
a hotspot in research of multiple attribute decision-making problems. Fan et al. [15] and Wang et al. [16], respectively, studied the multiple attribute decision-making problem of the real number with the alternative preference information as the complementary judgment matrix and the reciprocal judgment matrix. Xu and Chen $[17,18]$ studied the decisionmaking methods of the interval fuzzy preference relation, the interval utility value, and the interval multiplicative preference relation. In $[19,20]$, the solving method of the two-tuple linguistic variable multiple attribute decision-making based on the preference information was studied by the method of the aggregation operator and the granularity transformation. The research on the decision-making method with preference on alternatives, utilizing the fuzzy number and the intuitionistic fuzzy number to describe the attribute values, is a hot research field in recent years. Xu [21], using the similarity and the complementary judgment matrix ranking formula, solved the alternative preference problem whose attribute value is noted by triangular fuzzy numbers. Based on this, Liu and $\mathrm{He}$ [22] made further improvement and introduced the possibility degree formula for the alternative selection. Wei et al. [23] solved the problem of the intuitionistic fuzzy multiple attribute decision-making with preference on alternatives by establishing the goal programming model based on the minimum deviation and the score function ranking. In [24], a new method of constructing the distance optimization model based on the unification of the partial preference and the global preference of the decision-makers was proposed, which substituted the aggregated global preference into the objective function of the mathematical programming in the form of the intuitionistic fuzzy weighted averaging operator. Although the existing methods of the intuitionistic fuzzy multiple attribute decision-making are improved, the computation is relatively complex, and it is not suitable for the decision-making problem with many alternatives and the attributes.

At the same time, the concept and the theory of the cross entropy in information entropy are applied to the fuzzy multiple attribute decision-making. Shang and Jiang [25] gave the definition of the cross entropy of fuzzy sets in earlier times; then Vlachos and Sergiadis [26] extended it to the field of intuitionistic fuzzy sets. Because the cross entropy may not be calculated when the membership degree, the nonmembership degree, and the uncertainty degree of the intuitionistic fuzzy number take specific parameter values, Zhang and Jiang [27] and Ye [28] made further improvement. Xia and Xu [29] used the cross entropy to determine the weights of experts and the attributes of intuitionistic fuzzy group decisionmaking. Li [30] improved the interval valued intuitionistic fuzzy entropy by the continuous ordered weighted averaging operator (COWA) and combined it with the TOPSIS decision method to realize the optimum selection of the alternatives. Darvishi et al. [31] combined the intuitionistic fuzzy cross entropy with the principal component analysis method to solve the multiple attribute decision-making problem of a relatively large amount of information. So far, it has been found rarely that the cross entropy theory is applied to the multiple attribute decision-making problems with preference information on alternatives.
From the existing results, the research method of the intuitionistic fuzzy multiple attribute decision-making based on preference on alternatives is not perfect. Firstly, most of the existing decision models based on the deviation are the distance optimization model, while the existing intuitionistic fuzzy number distance measure formula has some defects, which often cannot distinguish the data size or the information confusion; secondly, the research is not deep enough for the unknown or partially unknown attribute weights, which often overlooks the case of the uncertainty of attribute weights. From the perspective of improving the accuracy of decision results, the intuitionistic fuzzy multiple attribute decision-making method based on preference on alternatives still needs further in-depth study.

In view of the above analysis, we propose a comprehensive decision model based on the intuitionistic fuzzy cross entropy distance and the grey correlation analysis to solve the problem of the intuitionistic fuzzy multiple attribute decision-making with preference on alternatives in this paper, which makes up the deficiency of causing the information confusion easily for the traditional intuitionistic fuzzy distance measure, improves the accuracy of the distance measure, solves the attribute weights by combining with the grey correlation analysis suitable for the small sample and the poor information decision-making, setting up the mathematical programming model with the maximum synthesis grey correlation coefficient between the evaluation value and the subjective preference value of the decision-makers, and then sorts the alternatives according to the change of the grey identification coefficients to demonstrate the stability. At last, the validity of the model is proved by the example of a venture capital firm.

\section{The Basic Theory of the Intuitionistic Fuzzy Set}

In this section, we introduce some basic knowledge and the necessary concepts related to the intuitionistic fuzzy set and the distance measure formula.

\subsection{The Intuitionistic Fuzzy Set}

Definition 1 (see [2]). Suppose $X$ is a nonempty set and the intuitionistic fuzzy set $A$ on the domain $X$ is defined as follows:

$$
A=\left\{\left\langle x, \mu_{A}(x), v_{A}(x)\right\rangle \mid x \in X\right\},
$$

where $\mu_{A}(x)$ and $v_{A}(x)$ denote, respectively, the degrees of membership and nonmembership where element $x$ belonged to $A$ on $X$ : that is, $\mu_{A}: X \rightarrow[0,1], x \in X \rightarrow \mu_{A}(x) \in[0,1]$, $v_{A}: X \rightarrow[0,1], x \in X \rightarrow v_{A}(x) \in[0,1]$, and $0 \leq \mu_{A}(x)+$ $\nu_{A}(x) \leq 1$; then $\pi_{A}=1-\mu_{A}(x)-v_{A}(x)$ is called the uncertainty degree where element $x$ belonged to $A$ on $X$. Obviously, for any $x \in X, 0 \leq \pi_{A}(x) \leq 1$. Some basic operations of intuitionistic fuzzy sets are shown in Definition 2.

Definition 2 (see [32]). If $\alpha=\left(\mu_{\alpha}, v_{\alpha}\right), \alpha_{1}=\left(\mu_{\alpha_{1}}, v_{\alpha_{1}}\right)$, and $\alpha_{2}=\left(\mu_{\alpha_{2}}, \nu_{\alpha_{2}}\right)$ are all intuitionistic fuzzy numbers, then

(1) $\bar{\alpha}=\left(\nu_{\alpha}, \mu_{\alpha}\right)$; 
(2) $\alpha_{1} \wedge \alpha_{2}=\left\{\min \left(\mu_{\alpha_{1}}, \mu_{\alpha_{2}}\right), \max \left(\nu_{\alpha_{1}}, \nu_{\alpha_{2}}\right)\right\}$;

(3) $\alpha_{1} \vee \alpha_{2}=\left\{\max \left(\mu_{\alpha_{1}}, \mu_{\alpha_{2}}\right), \min \left(\nu_{\alpha_{1}}, \nu_{\alpha_{2}}\right)\right\}$;

(4) $\alpha_{1} \oplus \alpha_{2}=\left(\mu_{\alpha_{1}}+\mu_{\alpha_{2}}-\mu_{\alpha_{1}} \mu_{\alpha_{2}}, \nu_{\alpha_{1}} \nu_{\alpha_{2}}\right)$;

(5) $\alpha_{1} \otimes \alpha_{2}=\left(\mu_{\alpha_{1}} \mu_{\alpha_{2}}, v_{\alpha_{1}}+\nu_{\alpha_{2}}-v_{\alpha_{1}} \nu_{\alpha_{2}}\right)$;

(6) $\lambda \alpha=\left(1-\left(1-\mu_{\alpha}\right)^{\lambda}, \nu_{\alpha}^{\lambda}\right), \lambda>0$;

(7) $\alpha^{\lambda}=\left(\mu_{\alpha}^{\lambda}, 1-\left(1-\nu_{\alpha}\right)^{\lambda}\right), \lambda>0$.

2.2. The Distance Measure Formula of Intuitionistic Fuzzy Set. Based on the geometric distance model, Xu [33] proposed a distance measure formula of the intuitionistic fuzzy set. The intuitionistic fuzzy integration operator is defined as follows.

Definition 3. Let $d$ be a mapping: $d:(\Phi(X))^{2} \rightarrow[0,1]$. If there are the intuitionistic fuzzy sets $A=\left\{\left\langle x, \mu_{A}(x), v_{A}(x)\right\rangle \mid\right.$ $x \in X\}$ and $B=\left\{\left\langle x, \mu_{B}(x), \nu_{B}(x)\right\rangle \mid x \in X\right\}$ then the distance measure formula is defined as follows:

$$
\begin{aligned}
& d_{X u}(A, B)=\left[\frac { 1 } { 2 n } \sum _ { j = 1 } ^ { n } \left(\left|\mu_{A}\left(x_{j}\right)-\mu_{B}\left(x_{j}\right)\right|^{\lambda}\right.\right. \\
& +\left|\nu_{A}\left(x_{j}\right)-v_{B}\left(x_{j}\right)\right|^{\lambda} \\
& \left.\left.+\left|\pi_{A}\left(x_{j}\right)-\pi_{B}\left(x_{j}\right)\right|^{\lambda}\right)\right]^{1 / \lambda} .
\end{aligned}
$$

When $\lambda=1, d_{X u}$ is reduced to the Hamming distance:

$$
\begin{aligned}
& d_{H}(A, B)=\frac{1}{2 n} \sum_{j=1}^{n}\left(\left|\mu_{A}\left(x_{j}\right)-\mu_{B}\left(x_{j}\right)\right|\right. \\
& \left.\quad+\left|\nu_{A}\left(x_{j}\right)-v_{B}\left(x_{j}\right)\right|+\left|\pi_{A}\left(x_{j}\right)-\pi_{B}\left(x_{j}\right)\right|\right) .
\end{aligned}
$$

When $\lambda=2, d_{X u}$ is reduced to the Euclidean distance:

$$
d_{E}(A, B)=\sqrt{\frac{1}{2 n} \sum_{j=1}^{n}\left(\left|\mu_{A}\left(x_{j}\right)-\mu_{B}\left(x_{j}\right)\right|^{2}+\left|\nu_{A}\left(x_{j}\right)-v_{B}\left(x_{j}\right)\right|^{2}+\left|\pi_{A}\left(x_{j}\right)-\pi_{B}\left(x_{j}\right)\right|^{2}\right)} .
$$

\section{Intuitionistic Fuzzy Cross Entropy}

Definition 4 (see [28]). Assume a domain $X=\left\{x_{1}, x_{2}, \ldots\right.$, $\left.x_{n}\right\}, A$ and $B$ are two intuitionistic fuzzy sets on $X, A=$ $\left\{\left\langle x_{i}, \mu_{A}\left(x_{i}\right), v_{A}\left(x_{i}\right)\right\rangle \mid x_{i} \in X\right\}, B=\left\{\left\langle x_{i}, \mu_{B}\left(x_{i}\right), v_{B}\left(x_{i}\right)\right\rangle \mid\right.$ $\left.x_{i} \in X\right\}$, and then the intuitionistic fuzzy cross entropy of $A$ and $B$ is

$$
\begin{aligned}
\operatorname{CE}(A, B) & =\sum_{i=1}^{n}\left(\frac{1+\mu_{A}\left(x_{i}\right)-v_{A}\left(x_{i}\right)}{2}\right. \\
\cdot & \left.\log _{2} \frac{1+\mu_{A}\left(x_{i}\right)-v_{A}\left(x_{i}\right)}{(1 / 2)\left[\left(1+\mu_{A}\left(x_{i}\right)-v_{A}\left(x_{i}\right)\right)+\left(1+\mu_{B}\left(x_{i}\right)-v_{B}\left(x_{i}\right)\right)\right]}\right) \\
+ & \sum_{i=1}^{n}\left(\frac{1-\mu_{A}\left(x_{i}\right)+v_{A}\left(x_{i}\right)}{2}\right. \\
\cdot & \left.\log _{2} \frac{1-\mu_{A}\left(x_{i}\right)+v_{A}\left(x_{i}\right)}{(1 / 2)\left[\left(1-\mu_{A}\left(x_{i}\right)+v_{A}\left(x_{i}\right)\right)+\left(1-\mu_{B}\left(x_{i}\right)+v_{B}\left(x_{i}\right)\right)\right]}\right) .
\end{aligned}
$$

The intuitionistic fuzzy cross entropy $\operatorname{CE}(A, B)$ does not satisfy the symmetry, so let

$$
\mathrm{CE}^{*}(A, B)=\mathrm{CE}(A, B)+\mathrm{CE}(B, A)
$$

be the improved form of the intuitionistic fuzzy cross entropy and define it as the intuitionistic fuzzy cross entropy distance.

Theorem 5. The intuitionistic fuzzy cross entropy distance $C E^{*}(A, B)$ satisfies the following properties:

(1) $C E^{*}(A, B) \geq 0$;

(2) If $A=B$, then $C E^{*}(A, B)=0$;
(3) $C E^{*}(A, B)=C E^{*}(\bar{A}, \bar{B})$;

(4) $C E^{*}(A, B)=C E^{*}(B, A)$.

Proof. (1) One has

$$
\begin{aligned}
& \operatorname{CE}(A, B)=\sum_{i=1}^{n}\left(\frac{1+\mu_{A}\left(x_{i}\right)-v_{A}\left(x_{i}\right)}{2}\right. \\
& \left.\cdot \log _{2} \frac{1+\mu_{A}\left(x_{i}\right)-v_{A}\left(x_{i}\right)}{(1 / 2)\left[\left(1+\mu_{A}\left(x_{i}\right)-v_{A}\left(x_{i}\right)\right)+\left(1+\mu_{B}\left(x_{i}\right)-v_{B}\left(x_{i}\right)\right)\right]}\right) \\
& +\sum_{i=1}^{n}\left(\frac{1-\mu_{A}\left(x_{i}\right)+v_{A}\left(x_{i}\right)}{2}\right. \\
& \left.\cdot \log _{2} \frac{1-\mu_{A}\left(x_{i}\right)+v_{A}\left(x_{i}\right)}{(1 / 2)\left[\left(1-\mu_{A}\left(x_{i}\right)+v_{A}\left(x_{i}\right)\right)+\left(1-\mu_{B}\left(x_{i}\right)+v_{B}\left(x_{i}\right)\right)\right]}\right),
\end{aligned}
$$

so

$$
\begin{aligned}
- & \operatorname{CE}(A, B)=-\sum_{i=1}^{n}\left(\frac{1+\mu_{A}\left(x_{i}\right)-v_{A}\left(x_{i}\right)}{2}\right. \\
& \left.\cdot \log _{2} \frac{1+\mu_{A}\left(x_{i}\right)-v_{A}\left(x_{i}\right)}{(1 / 2)\left[\left(1+\mu_{A}\left(x_{i}\right)-v_{A}\left(x_{i}\right)\right)+\left(1+\mu_{B}\left(x_{i}\right)-v_{B}\left(x_{i}\right)\right)\right]}\right) \\
& -\sum_{i=1}^{n}\left(\frac{1-\mu_{A}\left(x_{i}\right)+v_{A}\left(x_{i}\right)}{2}\right. \\
& \left.\cdot \log _{2} \frac{1-\mu_{A}\left(x_{i}\right)+v_{A}\left(x_{i}\right)}{(1 / 2)\left[\left(1-\mu_{A}\left(x_{i}\right)+v_{A}\left(x_{i}\right)\right)+\left(1-\mu_{B}\left(x_{i}\right)+v_{B}\left(x_{i}\right)\right)\right]}\right) \\
& =\sum_{i=1}^{n}\left(\frac{1+\mu_{A}\left(x_{i}\right)-v_{A}\left(x_{i}\right)}{2}\right.
\end{aligned}
$$




$$
\begin{aligned}
& \left.\cdot \log _{2} \frac{(1 / 2)\left[\left(1+\mu_{A}\left(x_{i}\right)-v_{A}\left(x_{i}\right)\right)+\left(1+\mu_{B}\left(x_{i}\right)-v_{B}\left(x_{i}\right)\right)\right]}{1+\mu_{A}\left(x_{i}\right)-v_{A}\left(x_{i}\right)}\right) \\
& +\sum_{i=1}^{n}\left(\frac{1-\mu_{A}\left(x_{i}\right)+v_{A}\left(x_{i}\right)}{2}\right. \\
& \left.\cdot \log _{2} \frac{(1 / 2)\left[\left(1-\mu_{A}\left(x_{i}\right)+v_{A}\left(x_{i}\right)\right)+\left(1-\mu_{B}\left(x_{i}\right)+v_{B}\left(x_{i}\right)\right)\right]}{1-\mu_{A}\left(x_{i}\right)+v_{A}\left(x_{i}\right)}\right) .
\end{aligned}
$$

According to Jansen's inequality [34], if $f(x)$ is strictly convex, then

$$
\begin{aligned}
& f\left(a_{1} x_{1}+a_{2} x_{2}+\cdots+a_{n} x_{n}\right) \\
& \quad \leq a_{1} f\left(x_{1}\right)+a_{2} f\left(x_{2}\right)+\cdots+a_{n} f\left(x_{n}\right) .
\end{aligned}
$$

Because the logarithmic function above is strictly convex, then

$$
\begin{aligned}
-\operatorname{CE}(A, B) \leq & \sum_{i=1}^{n} \log _{2}\left(\frac{1+\mu_{A}\left(x_{i}\right)-v_{A}\left(x_{i}\right)}{2} \frac{(1 / 2)\left[\left(1+\mu_{A}\left(x_{i}\right)-v_{A}\left(x_{i}\right)\right)+\left(1+\mu_{B}\left(x_{i}\right)-v_{B}\left(x_{i}\right)\right)\right]}{1+\mu_{A}\left(x_{i}\right)-v_{A}\left(x_{i}\right)}\right) \\
& +\sum_{i=1}^{n} \log _{2}\left(\frac{1-\mu_{A}\left(x_{i}\right)+v_{A}\left(x_{i}\right)}{2} \frac{(1 / 2)\left[\left(1-\mu_{A}\left(x_{i}\right)+v_{A}\left(x_{i}\right)\right)+\left(1-\mu_{B}\left(x_{i}\right)+v_{B}\left(x_{i}\right)\right)\right]}{1-\mu_{A}\left(x_{i}\right)+v_{A}\left(x_{i}\right)}\right) \\
\leq & \log _{2} \frac{\left(1+\mu_{A}\left(x_{i}\right)-v_{A}\left(x_{i}\right)\right)+\left(1+\mu_{B}\left(x_{i}\right)-v_{B}\left(x_{i}\right)\right)+\left(1-\mu_{A}\left(x_{i}\right)+v_{A}\left(x_{i}\right)\right)+\left(1-\mu_{B}\left(x_{i}\right)+v_{B}\left(x_{i}\right)\right)}{4} \\
= & 0,
\end{aligned}
$$

so $\mathrm{CE}(A, B) \geq 0$. The same can be proved where $\mathrm{CE}(B, A) \geq$ 0 , so $\mathrm{CE}^{*}(A, B) \geq 0$.

(2) When $A=B$, obviously, $\mu_{A}\left(x_{i}\right)=\mu_{B}\left(x_{i}\right), v_{A}\left(x_{i}\right)=$ $\nu_{B}\left(x_{i}\right)$, substitute them into formulas (5) and (6), and $\mathrm{CE}^{*}(A$, $B)=0$.

(3) As can be seen from the complementation of the intuitionistic fuzzy sets, $\bar{A}=\left\{\left\langle x_{i}, v_{A}\left(x_{i}\right), \mu_{A}\left(x_{i}\right)\right\rangle\right\}, \bar{B}=\left\{\left\langle x_{i}\right.\right.$, $\left.\left.\nu_{B}\left(x_{i}\right), \mu_{B}\left(x_{i}\right)\right\rangle\right\}$, substitute them into formulas (5) and (6), and obviously $\mathrm{CE}^{*}(A, B)=\mathrm{CE}^{*}(\bar{A}, \bar{B})$.

(4) Because $\operatorname{CE}^{*}(A, B)$ satisfies the symmetry, the exchange of intuitionistic fuzzy sets $A$ and $B$ does not affect the entropy result: that is, $\mathrm{CE}^{*}(A, B)=\mathrm{CE}^{*}(B, A)$.

As can be seen from property (2), when the two intuitionistic fuzzy sets are exactly equal, the intuitionistic fuzzy cross entropy is the least; therefore, the cross entropy can be used to measure the difference between two intuitionistic fuzzy sets. The intuitionistic fuzzy cross entropy adds the meaning of the information entropy on the basis of the original intuitionistic fuzzy complete information. It can be used to measure the fuzzy degree and the uncertainty degree of the intuitionistic fuzzy sets. The greater the cross entropy of two intuitionistic fuzzy numbers, the further the distance [26]. The following example is proposed to prove that the intuitionistic fuzzy entropy can better reflect the difference between the data than the traditional intuitionistic fuzzy distance measure formula.

For example, there are three intuitionistic fuzzy numbers $\alpha_{1}=(0.4,0.3), \alpha_{2}=(0.5,0.3)$, and $\alpha_{3}=(0.5,0.2)$. With the Hamming distance formula (3), there is $d_{H}\left(\alpha_{1}, \alpha_{3}\right)=$ $d_{H}\left(\alpha_{2}, \alpha_{3}\right)$. With the Euclidean formula distance formula (4), there is $d_{E}\left(\alpha_{1}, \alpha_{3}\right)=d_{E}\left(\alpha_{2}, \alpha_{3}\right)$. Obviously, it is difficult to compare the two distances. Compute, with formula (6), $\operatorname{CE}^{*}\left(\alpha_{1}, \alpha_{3}\right)=0.0151$ and $\operatorname{CE}^{*}\left(\alpha_{2}, \alpha_{3}\right)=0.0038$. Comparing with $\alpha_{1}, \alpha_{2}$ is closer to $\alpha_{3}$, which is consistent with the people's intuition.

\section{Multiple Attribute Decision-Making Method Based on Intuitionistic Fuzzy Cross Entropy}

4.1. Problem Description. In this paper, the multiple attribute decision-making problems are assumed to have a certain subjective preference for the decision-makers. Generally the problems can be abstracted as follows: the decision-makers can give the attribute values in the form of the intuitionistic fuzzy numbers $\left(\mu_{i j}, v_{i j}\right)$ from the alternatives $A_{i}(i=$ $1,2, \ldots, m)$ according to the evaluation attributes $C_{j}(j=$ $1,2, \ldots, n)$, where $\mu_{i j}$ denotes the approval degree about $A_{i}$ under $C_{j}, v_{i j}$ denotes the disapproval degree about $A_{i}$ under $C_{j}, \pi_{i j}=1-\mu_{i j}-v_{i j}$ denotes the uncertainty degree, $0 \leq \mu_{i j} \leq$ $1,0 \leq v_{i j} \leq 1,0 \leq \pi_{i j} \leq 1$, and $\omega_{j}(j=1,2, \ldots, n)$ denotes the attribute weights; meanwhile, $\sum_{j=1}^{n} \omega_{j}=1$. The intuitionistic fuzzy decision matrix $R_{m n}$ is shown in Table 1.

Suppose the decision-makers have some preference on alternatives $A_{i}(i=1,2, \ldots, m)$ and the preference values are noted by the intuitionistic fuzzy numbers $o_{i}=\left(\alpha_{i}, \beta_{i}\right)(i=$ $1,2, \ldots, m)$; then we adopt the optimization model based on the intuitionistic fuzzy cross entropy and the grey correlation analysis method to find the optimum solution among the alternatives with preference.

4.2. Decision Method and Steps. In this paper, the intuitionistic fuzzy multiple attribute decision-making method with preference on alternatives draws lessons from the theory of the intuitionistic fuzzy cross entropy distance and the grey correlation analysis method to solve the optimum alternative selection problem in the case where the weights are partly unknown.

Step 1. Determine the alternatives $A_{i}(i=1,2, \ldots, m)$, the evaluation attributes $C_{j}(j=1,2, \ldots, n)$, the subjective evaluation matrix of the decision-makers $R_{m n}$, and the subjective preference $o_{i}=\left(\alpha_{i}, \beta_{i}\right)$. 
TABLE 1: Intuitionistic fuzzy decision matrix $R_{m n}$.

\begin{tabular}{lcclc}
\hline & $C_{1}$ & $C_{2}$ & $\ldots$ & $C_{n}$ \\
\hline$A_{1}$ & $\left(\mu_{11}, v_{11}\right)$ & $\left(\mu_{12}, v_{12}\right)$ & $\ldots$ & $\left(\mu_{1 n}, v_{1 n}\right)$ \\
$A_{2}$ & $\left(\mu_{21}, v_{21}\right)$ & $\left(\mu_{22}, v_{22}\right)$ & $\ldots$ & $\left(\mu_{2 n}, v_{2 n}\right)$ \\
$\vdots$ & $\vdots$ & $\vdots$ & $\ldots$ & $\vdots$ \\
$A_{m}$ & $\left(\mu_{m 1}, v_{m 1}\right)$ & $\left(\mu_{m 2}, v_{m 2}\right)$ & $\ldots$ & $\left(\mu_{m n}, v_{m n}\right)$ \\
\hline
\end{tabular}

Step 2. Calculate the grey correlation coefficient between the subjective evaluation of each alternative based on the intuitionistic fuzzy cross entropy distance and the subjective preference of the decision-makers. The formula is as follows:

$$
\gamma_{i j}=\frac{\min _{i} \min _{j} \mathrm{CE}_{i j}^{*}+\xi \max _{i} \max _{j} \mathrm{CE}_{i j}^{*}}{\mathrm{CE}_{i j}^{*}+\xi \max _{i} \max _{j} \mathrm{CE}_{i j}^{*}} .
$$

The grey correlation coefficient here shows the approximation degree of the subjective evaluation to the subjective preference of each $A_{i}$ under $C_{j}$. The greater the grey correlation coefficient $\gamma_{i j}$, the higher the approximation degree, and vice versa. In the formula above, $\mathrm{CE}_{i j}^{*}$ is the intuitionistic fuzzy cross entropy distance, and its formula is as follows:

$$
\begin{aligned}
& \mathrm{CE}_{i j}^{*}=\frac{1+\mu_{i j}-v_{i j}}{2} \\
& \cdot \log _{2} \frac{1+\mu_{i j}-v_{i j}}{(1 / 2)\left[\left(1+\mu_{i j}-v_{i j}\right)+\left(1+\alpha_{i}-\beta_{i}\right)\right]} \\
&+\frac{1-\mu_{i j}+v_{i j}}{2} \\
& \cdot \log _{2} \frac{1-\mu_{i j}+v_{i j}}{(1 / 2)\left[\left(1-\mu_{i j}+v_{i j}\right)+\left(1-\alpha_{i}+\beta_{i}\right)\right]} \\
&+ \frac{1+\alpha_{i}-\beta_{i}}{2} \quad 1+\alpha_{i}-\beta_{i} \\
& \cdot \log _{2} \frac{1}{(1 / 2)\left[\left(1+\alpha_{i}-\beta_{i}\right)+\left(1+\mu_{i j}-v_{i j}\right)\right]} \\
&+\frac{1-\alpha_{i}+\beta_{i}}{2} \quad 1-\alpha_{i}+\beta_{i} \\
& \cdot \log _{2} \frac{(1 / 2)\left[\left(1-\alpha_{i}+\beta_{i}\right)+\left(1-\mu_{i j}+v_{i j}\right)\right]}{(1)}
\end{aligned}
$$

In formula (11), $\xi$ is called the distinguishing coefficient: usually $\xi \in(0,1)$.

Step 3. The actual meaning has been clear by last step; therefore, each attribute weight can be determined by constructing the mathematical programming model with the purpose of maximizing the grey correlation coefficient.

Let the attribute weight be $\omega_{j}(j=1,2, \ldots, n)$; meanwhile, $\sum_{j=1}^{n} \omega_{j}=1$. Calculate the synthesis grey correlation coefficient of $A_{i}$ under each attribute using the following formula:

$$
\gamma_{i}=\sum_{j=1}^{n} \gamma_{i j} \omega_{j}, \quad(i=1,2, \ldots, m),(j=1,2, \ldots, n) .
$$

If the attribute weight $\omega_{j}$ is known, then sort the alternatives according to the grey correlation coefficient calculated by formula (11). The greater $\gamma_{i}$, the higher the approximation of the subjective evaluation to the subjective preference of alternative $A_{i}$, and, therefore, the higher in the rankings. However, in the actual decision-making process, because of the lack of knowledge and the complexity of the objective things, it is often difficult to determine the attribute weights for the decision-makers. In most cases, the attribute weights are partially unknown or even completely unknown. Consequently, how to determine the attribute weights is a key problem in the decision-making. In order to guarantee the feasibility and the validity of the decision-making method, the establishment of the weight should be based on the maximum similarity between the subjective evaluation and the subjective preference value of the alternative $A_{i}$. Therefore, the mathematical programming models can be established as

$$
\begin{array}{ll}
\max & Z\left(\omega_{j}\right)=\sum_{j=1}^{n} \gamma_{i j} \omega_{j} \\
\text { s.t. } & \sum_{j=1}^{n} \omega_{j}=1, \quad \omega_{j} \in W \\
& \omega_{j} \geq 0, \\
& j=1,2, \ldots, n, \\
& i=1,2, \ldots, m .
\end{array}
$$

(Model 1)

Note that s.t. is short for "subject to" (the same as below).

Since there is no preference relationship between the various alternatives, it is fair to compete, so (Model 1) can be transformed into a single objective optimization model as follows:

$$
\begin{array}{ll}
\max & Z\left(\omega_{j}\right)=\sum_{i=1}^{m} \sum_{j=1}^{n} \gamma_{i j} \omega_{j} \\
\text { s.t. } & \sum_{j=1}^{n} \omega_{j}=1, \quad \omega_{j} \in W \\
& \omega_{j} \geq 0, \\
& j=1,2, \ldots, n, \\
& i=1,2, \ldots, m .
\end{array}
$$

The attribute weights can be obtained by using the software MATLAB_R2014a. If the attribute weight part is known, then $\omega_{j} \in W$ and usually it can be used as a constraint condition in the mathematical programming in 5 cases as follows [35]:

(1) $\omega_{k} \geq \omega_{t}$ 
TABLE 2: The intuitionistic fuzzy decision matrix $R_{54}$.

\begin{tabular}{lcccc}
\hline & $C_{1}$ & $C_{2}$ & $C_{3}$ & $C_{4}$ \\
\hline$A_{1}$ & $(0.4,0.5)$ & $(0.5,0.4)$ & $(0.2,0.7)$ & $(0.3,0.5)$ \\
$A_{2}$ & $(0.7,0.2)$ & $(0.5,0.4)$ & $(0.2,0.5)$ & $(0.1,0.7)$ \\
$A_{3}$ & $(0.5,0.3)$ & $(0.3,0.4)$ & $(0.6,0.2)$ & $(0.4,0.4)$ \\
$A_{4}$ & $(0.6,0.4)$ & $(0.6,0.3)$ & $(0.6,0.3)$ & $(0.3,0.6)$ \\
$A_{5}$ & $(0.5,0.5)$ & $(0.4,0.5)$ & $(0.4,0.4)$ & $(0.5,0.4)$ \\
\hline
\end{tabular}

(2) $\omega_{k}-\omega_{t} \geq c, c>0$

(3) $\omega_{k} \geq c \omega_{t}, c>0$;

(4) $c \leq \omega_{i} \leq c+\tau, c>0, \tau>0$;

(5) $\omega_{k}-\omega_{t} \geq \omega_{l}-\omega_{e}, t \neq l \neq e$.

Step 4. Substitute the obtained attribute weight $\omega_{j}$ into formula (13) and then calculate the grey correlation coefficient $\gamma_{i}$ of the alternative $A_{i}$ under all the attributes.

Step 5. Sort the alternatives by the synthesis grey correlation coefficient $\gamma_{i}$ of each alternative. The greater $\gamma_{i}$, the better the alternative.

Step 6. Make the perturbation analysis according to the variation of the distinguishing coefficient $\xi$ in the formula of the grey correlation coefficient (11) in order to test the stability and the reliability of the method.

\section{Example Analysis}

In order to prove the accuracy and the validity of the method in this chapter, we use the example in document [24] for calculation and analysis. A venture capital firm intends to make evaluation and selection to 5 enterprises with the investment potential: $A_{1}$ : automobile company, $A_{2}$ : military manufacturing enterprise, $A_{3}$ : TV media company, $A_{4}$ : food enterprises, and $A_{5}$ : computer software company. The 5 enterprises are evaluated under four conditions including the social and the political factors $\left(C_{1}\right)$, the environmental factors $\left(C_{2}\right)$, the investment risk factors $\left(C_{3}\right)$, and the enterprise growth factors $\left(C_{4}\right)$. The evaluation values are noted in the form of the intuitionistic fuzzy numbers and the obtained intuitionistic fuzzy decision matrix $R_{54}$ is shown in Table 2 .

The subjective preference of the decision-makers on alternatives $A_{i}(i=1,2, \ldots, 5)$ is also noted by the intuitionistic fuzzy numbers: that is, $o_{1}=(0.3,0.5), o_{2}=(0.7,0.2), o_{3}=$ $(0.4,0.3), o_{4}=(0.6,0.2)$, and $o_{5}=(0.5,0.4)$, and the known weights satisfy $0.25 \leq \omega_{1} \leq 0.28,0.20 \leq \omega_{2} \leq 0.25$, $0.22 \leq \omega_{3} \leq 0.25$, and $0.25 \leq \omega_{4} \leq 0.30$. In order to select the optimal target investment enterprise, the venture capital firm adopts the decision-making method constructed in this paper.

Specific calculation steps are as follows.

Step 1. Determine the alternatives $A_{i}(i=1,2, \ldots, 5)$, the evaluation attributes $C_{j}(j=1,2, \ldots, 4)$, the subjective evaluation matrix $R_{54}$, and the subjective preference $o_{i}=$ $\left(\alpha_{i}, \beta_{i}\right)(i=1,2, \ldots, 5)$, as shown in Table 2 .
Step 2. Calculate the intuitionistic fuzzy cross entropy distances between the subjective evaluation and the subjective preference of each alternative and form the distance matrix $\mathrm{CE}^{*}$ as follows:

$$
\mathrm{CE}^{*}=\mathrm{CE}_{54}^{*}=\left[\begin{array}{llll}
0.0037 & 0.0327 & 0.0372 & 0.0000 \\
0.0000 & 0.0641 & 0.2402 & 0.4632 \\
0.0037 & 0.0145 & 0.0348 & 0.0036 \\
0.0159 & 0.0041 & 0.0041 & 0.1810 \\
0.0036 & 0.0145 & 0.0036 & 0.0000
\end{array}\right]
$$

Then calculate the grey correlation coefficient between the subjective evaluation and the subjective preference of each alternative. Suppose $\xi=0.5$, and the obtained coefficient matrix $\gamma$ is shown as follows:

$$
\gamma=\gamma_{54}=\left[\begin{array}{llll}
0.9843 & 0.8763 & 0.8616 & 1.0000 \\
1.0000 & 0.7832 & 0.4909 & 0.3333 \\
0.9843 & 0.9411 & 0.8694 & 0.9847 \\
0.9358 & 0.9826 & 0.9826 & 0.5613 \\
0.9847 & 0.9411 & 0.9847 & 1.0000
\end{array}\right] .
$$

Step 3. Construct the mathematical programming model with the goal of maximizing the grey correlation coefficient:

$$
\begin{array}{ll}
\max \quad Z\left(\omega_{j}\right) \\
=4.8991 \omega_{1}+4.5243 \omega_{2}+4.1892 \omega_{3} \\
\quad+3.8793 \omega_{4} \\
\text { s.t. } \quad 0.25 \leq \omega_{1} \leq 0.28 \\
0.20 \leq \omega_{2} \leq 0.25 \\
0.22 \leq \omega_{3} \leq 0.25 \\
0.25 \leq \omega_{4} \leq 0.30 \\
\omega_{1}+\omega_{2}+\omega_{3}+\omega_{4}=1 \\
\omega_{j} \geq 0, \quad j=1,2,3,4 .
\end{array}
$$

We performed calculation by MATLAB_R2014a and the obtained attribute weight values are $\omega_{1}=0.28, \omega_{2}=0.25$, $\omega_{3}=0.22$, and $\omega_{4}=0.25$.

Step 4. Substitute the obtained attribute weight $\omega_{j}$ into formula (13) and calculate the synthesis grey correlation coefficient $\gamma_{i}(i=1,2, \ldots, 5)$ under all the attributes:

$$
\begin{aligned}
& \gamma_{1}=0.9342 \\
& \gamma_{2}=0.6671 ; \\
& \gamma_{3}=0.9483 ; \\
& \gamma_{4}=0.8642 ; \\
& \gamma_{5}=0.9776
\end{aligned}
$$


TABLE 3: The attribute weight values under different grey resolutions.

\begin{tabular}{cccccccc}
\hline & $\xi=0.05$ & $\xi=0.20$ & $\xi=0.35$ & $\xi=0.50$ & $\xi=0.65$ & $\xi=0.80$ & \multicolumn{2}{c}{$\xi=0.95$} \\
\hline$\omega_{1}$ & 0.28 & 0.28 & 0.28 & 0.28 & 0.28 & 0.28 \\
$\omega_{2}$ & 0.20 & 0.25 & 0.25 & 0.25 & 0.25 & 0.25 & 0.25 \\
$\omega_{3}$ & 0.22 & 0.22 & 0.22 & 0.22 & 0.22 & 0.22 & 0.22 \\
$\omega_{4}$ & 0.30 & 0.25 & 0.25 & 0.25 & 0.25 & 0.25 & 0.25 \\
\hline
\end{tabular}

TABLE 4: The decision-making results under different grey resolutions.

\begin{tabular}{cccccccccccccccc}
\hline & \multicolumn{2}{c}{$\xi=0.05$} & \multicolumn{2}{c}{$\xi=0.20$} & \multicolumn{2}{c}{$\xi=0.35$} & \multicolumn{2}{c}{$\xi=0.50$} & \multicolumn{2}{c}{$\xi=0.65$} & \multicolumn{2}{c}{$\xi=0.80$} \\
& $\gamma_{i}$ & Rank & $\gamma_{i}$ & Rank & $\gamma_{i}$ & Rank & $\gamma_{i}$ & Rank & $\gamma_{i}$ & Rank & $\gamma_{i}$ & Rank & $\gamma_{i}$ & Rank \\
\hline$A_{1}$ & 0.70 & 3 & 0.86 & 3 & 0.91 & 3 & 0.93 & 3 & 0.95 & 3 & 0.96 & 3 & 0.96 & 3 \\
$A_{2}$ & 0.37 & 5 & 0.53 & 5 & 0.61 & 5 & 0.67 & 5 & 0.71 & 5 & 0.74 & 5 & 0.76 & 5 \\
$A_{3}$ & 0.71 & 2 & 0.89 & 2 & 0.93 & 2 & 0.95 & 2 & 0.96 & 2 & 0.97 & 2 & 0.97 & 2 \\
$A_{4}$ & 0.56 & 4 & 0.77 & 4 & 0.83 & 4 & 0.86 & 4 & 0.89 & 4 & 0.90 & 4 & 0.91 & 4 \\
$A_{5}$ & 0.86 & 1 & 0.95 & 1 & 0.97 & 1 & 0.98 & 1 & 0.98 & 1 & 0.99 & 1 & 0.99 & 1 \\
\hline
\end{tabular}

Step 5. Sort the alternatives by the synthesis grey correlation coefficient $\gamma_{i}(i=1,2, \ldots, 5), \gamma_{5} \geq \gamma_{3} \geq \gamma_{1} \geq \gamma_{4} \geq \gamma_{2}$, so $A_{5}>A_{3}>A_{1}>A_{4}>A_{2} \cdot \gamma_{3}$ is the greatest, so $A_{3}$ is the optimum: that is, the target the venture capital firm selects to invest is the computer software company.

Step 6. Make the perturbation analysis according to the variation of the distinguishing coefficient $\xi$ in the formula of grey correlation coefficient (5). Let $\xi=0.05,0.20,0.35,0.50,0.65$, 080 , and 0.95 . The attribute weights $\omega_{j}(j=1,2, \ldots, 4)$, the synthesis grey correlation coefficients $\gamma_{i}(i=1,2, \ldots, 5)$, and the ranking of the alternatives are shown in Tables 3 and 4 .

As can be seen from Table 4, although the grey distinguishing coefficient $\xi$ is assigned 7 dispersed values, the decision result does not vary with the change of the grey distinguishing coefficient, and the ranking result still keeps the same, $A_{5}>A_{3}>A_{1}>A_{4}>A_{2}$, which can demonstrate that the method constructed in this paper is stable and reliable.

However, the result of this paper is difficult compared to that of [24] whose ranking result is $A_{5}>A_{1}>A_{3}>$ $A_{4}>A_{2}$. The investment target the venture capital enterprise selects is still the computer software company, but the ranking of the other alternatives is different. The main reasons lie in the following. (1) The distance measure of the intuitionistic fuzzy multiple attribute decision-making method with preference information on alternatives adopts the intuitionistic fuzzy cross entropy, which is not like the geometric distance such as the Hamming or the Euclidean distance, but a distance of information that can better reflect the difference between the intuitionistic fuzzy sets and not just keep the information. Take the calculation process as an example in this section. The subjective preference on alternative $A_{3}$ is $o_{3}=(0.4,0.3)$, but in the initial decision matrix, the subjective evaluation values under attributes $C_{1}$ and $C_{2}$ are given $(0.5,0.3)$ and $(0.3,0.4)$ by the decision-makers. When calculating with the Hamming or the Euclidean distance formula, the two distances between the evaluation value and the preference value are the same, difficult to distinguish, so the accuracy of the decision result will be affected. However, with the intuitionistic fuzzy cross entropy distance proposed in this section, the distances are 0.0037 and 0.0145 , making it easy to distinguish and forming good conditions for improving the accuracy of the decision result. (2) The grey correlation analysis method used in this section is suitable for the small sample and the poor information problem. The specific steps include applying it in the intuitionistic fuzzy multiple attribute decision-making, making full use of the known decision information such as the membership degree, the nonmembership degree, and the uncertainty degree, and then carrying out the perturbation analysis according to the change of the grey distinguishing coefficient. It turns out that combining the grey correlation analysis method with the intuitionistic fuzzy multiple attribute decision-making is suitable for the small sample decision-making; moreover, the effectiveness and the stability of the decision results are both relatively high.

\section{Conclusion}

In this paper, a comprehensive decision model based on the intuitionistic fuzzy cross entropy distance and the grey correlation analysis is proposed for the multiple attribute decision-making problems with the attribute weights partially unknown, the evaluation information in the form of intuitionistic fuzzy numbers, and the preference information on alternatives. The model introduces the intuitionistic fuzzy cross entropy distance to substitute the traditional geometric distance, calculates the attribute weights by constructing the mathematical optimization model of maximizing the synthesis grey correlation coefficient between the decision-making evaluation values and decision-makers' subjective preference values and making use of the known partial weight information, then sorts the alternatives by the synthesis grey correlation coefficient values, and at last demonstrates the effectiveness of the proposed model through the comparison and the analysis of two calculation examples. In this paper, the model 
has strong pertinence, high accuracy, and simple calculation and has further perfected and enriched the intuitionistic fuzzy multiple attribute decision-making theory. In future research, we will focus on the decision model and method constructed in this paper. Comparing to the study of alternatives with the definite preference, this research field will be more complex and more innovative in the multiple attribute decision-making method.

\section{Conflict of Interests}

The authors declare that there is no conflict of interests regarding the publication of this paper.

\section{Acknowledgments}

This paper was supported by the National Natural Science Foundation of China (71271070), Specialty of College Comprehensive Reform Pilot Project (ZG0429), and Specialty and Curriculum Integration Project of Guangxi High School (GXTSZY016).

\section{References}

[1] L. A. Zadeh, "Fuzzy sets," Information and Control, vol. 8, no. 3, pp. 338-353, 1965.

[2] K. T. Atanassov, "Intuitionistic fuzzy sets," Fuzzy Sets and Systems, vol. 20, no. 1, pp. 87-96, 1986.

[3] K. Atanassov and G. Gargov, "Interval valued intuitionistic fuzzy sets," Fuzzy Sets and Systems, vol. 31, no. 3, pp. 343-349, 1989.

[4] E. Szmidt and J. Kacprzyk, "A similarity measure for intuitionistic fuzzy sets and its application in supporting medical diagnostic reasoning," in Artificial Intelligence and Soft ComputingICAISC 2004, vol. 3070 of Lecture Notes in Computer Science, pp. 388-393, Springer, Berlin, Germany, 2004.

[5] E. Szmidt and J. Kacprzyk, "A new concept of a similarity measure for intuitionistic fuzzy sets and its use in group decision making," Modeling Decisions for Artificial Intelligence, vol. 35, no. 8, pp. 272-282, 2005.

[6] Z. S. Xu, "Models for multiple attribute decision making with intuitionistic fuzzy information," International Journal of Uncertainty, Fuzziness and Knowledge-Based Systems, vol. 15, no. 3, pp. 285-297, 2007.

[7] G.-W. Wei, "Maximizing deviation method for multiple attribute decision making in intuitionistic fuzzy setting," KnowledgeBased Systems, vol. 21, no. 8, pp. 833-836, 2008.

[8] Z.-J. Wang, "Derivation of intuitionistic fuzzy weights based on intuitionistic fuzzy preference relations," Applied Mathematical Modelling, vol. 37, no. 9, pp. 6377-6388, 2013.

[9] D.-F. Li, “TOPSIS-based nonlinear-programming methodology for multiattribute decision making with interval-valued intuitionistic fuzzy sets," IEEE Transactions on Fuzzy Systems, vol. 18, no. 2, pp. 299-311, 2010.

[10] G. W. Wei, "Maximizing deviation method for multiple attribute decision making in intuitionistic fuzzy setting," KnowledgeBased Systems, vol. 21, no. 8, pp. 833-836, 2008.

[11] D.-F. Li, "Linear programming method for MADM with interval-valued intuitionistic fuzzy sets," Expert Systems with Applications, vol. 37, no. 8, pp. 5939-5945, 2010.
[12] H. M. Zhang and L. Y. Yu, "MADM method based on crossentropy and extended TOPSIS with interval-valued intuitionistic fuzzy sets," Knowledge-Based Systems, vol. 30, no. 2, pp. 115$120,2012$.

[13] H. M. Zhang, "Linguistic intuitionistic fuzzy sets and application in MAGDM," Journal of Applied Mathematics, vol. 2014, Article ID 432092, 11 pages, 2014.

[14] T.-Y. Chen, "A comparative analysis of score functions for multiple criteria decision making in intuitionistic fuzzy settings," Information Sciences, vol. 181, no. 17, pp. 3652-3676, 2011.

[15] Z.-P. Fan, J. Ma, and Q. Zhang, "An approach to multiple attribute decision making based on fuzzy preference information on alternatives," Fuzzy Sets and Systems, vol. 131, no. 1, pp. 101-106, 2002.

[16] P. Wang, Y. Li, Y. H. Wang, and Z. Q. Zhu, "A new method based on TOPSIS and response surface method for MCDM problems with interval numbers," Mathematical Problems in Engineering, vol. 2015, Article ID 938535, 11 pages, 2015.

[17] Z. S. Xu, "Multiple-attribute group decision making with different formats of preference information on attributes," IEEE Transactions on Systems, Man, and Cybernetics, Part B: Cybernetics, vol. 37, no. 6, pp. 1500-1511, 2007.

[18] Z. S. Xu and J. Chen, "MAGDM linear-programming models with distinct uncertain preference structures," IEEE Transactions on Systems, Man, and Cybernetics Part B: Cybernetics, vol. 38, no. 5, pp. 1356-1370, 2008.

[19] S. Alonso, F. J. Cabrerizo, F. Chiclana, F. Herrera, and E. HerreraViedma, "Group decision making with incomplete fuzzy linguistic preference relations," International Journal of Intelligent Systems, vol. 24, no. 2, pp. 201-222, 2009.

[20] G. Q. Zhang, Y. C. Dong, and Y. F. Xu, "Consistency and consensus measures for linguistic preference relations based on distribution assessments," Information Fusion, vol. 17, no. 1, pp. 46$55,2014$.

[21] Z. S. Xu, "Study on method for triangular fuzzy number-based multi-attribute decision making with preference information on alternatives," Systems Engineering and Electronics, vol. 24, no. 8, pp. 9-12, 20, 2002.

[22] W. F. Liu and X. He, "A triangular fuzzy number multi-attribute decision-making method with preference information on alternatives," Fuzzy Systems and Mathematics, vol. 27, no. 2, pp. 155161, 2013.

[23] G. W. Wei, H. J. Wang, R. Lin, and X. F. Zhao, "Grey relational analysis method for intuitionistic fuzzy multiple attribute decision making with preference information on alternatives," International Journal of Computational Intelligence Systems, vol. 4, no. 2, pp. 164-173, 2011.

[24] X. He and W. F. Liu, "An intuitionistic fuzzy multi-attribute decision-making method with preference on alternatives," Operations Research and Management Science, vol. 22, no. 1, pp. 36-40, 2013.

[25] X.-G. Shang and W.-S. Jiang, "A note on fuzzy information measures," Pattern Recognition Letters, vol. 18, no. 5, pp. 425432, 1997.

[26] I. K. Vlachos and G. D. Sergiadis, "Intuitionistic fuzzy information-applications to pattern recognition," Pattern Recognition Letters, vol. 28, no. 2, pp. 197-206, 2007.

[27] Q.-S. Zhang and S.-Y. Jiang, "A note on information entropy measures for vague sets and its applications," Information Sciences, vol. 178, no. 21, pp. 4184-4191, 2008. 
[28] J. Ye, "Fuzzy cross entropy of interval-valued intuitionistic fuzzy sets and its optimal decision-making method based on the weights of alternatives," Expert Systems with Applications, vol. 38, no. 5, pp. 6179-6183, 2011.

[29] M. M. Xia and Z. S. Xu, "Entropy/cross entropy-based group decision making under intuitionistic fuzzy environment," Information Fusion, vol. 13, no. 1, pp. 31-47, 2012.

[30] X. Y. Li, "Interval-valued intuitionistic fuzzy continuous crossentropy and its application in multi-attribute decision-making," Computer Engineering and Applications, vol. 49, no. 15, pp. 234237, 2013.

[31] S. Darvishi, A. Fatemi, and P. Faroughi, "Introduce a novel PCA method for intuitionistic fuzzy sets based on cross entropy," Applied Mathematics, vol. 6, no. 6, pp. 990-995, 2015.

[32] K. T. Atanassov, "New operations defined over the intuitionistic fuzzy sets," Fuzzy Sets and Systems, vol. 61, no. 2, pp. 137-142, 1994.

[33] Z. S. Xu, "Some similarity measures of intuitionistic fuzzy sets and their applications to multiple attribute decision making," Fuzzy Optimization and Decision Making, vol. 6, no. 2, pp. 109121, 2007.

[34] M. A. Khan, G. A. Khan, T. Ali, and A. Kilicman, "On the refinement of Jensen's inequality," Applied Mathematics and Computation, vol. 262, no. 1, pp. 128-135, 2015.

[35] S. H. Kim and B. S. Ahn, "Interactive group decision making procedure under incomplete information," European Journal of Operational Research, vol. 116, no. 3, pp. 498-507, 1999. 


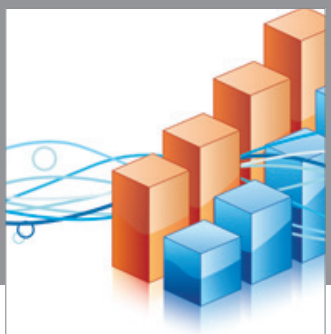

Advances in

Operations Research

vatem alat4

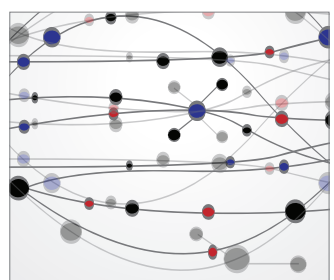

\section{The Scientific} World Journal
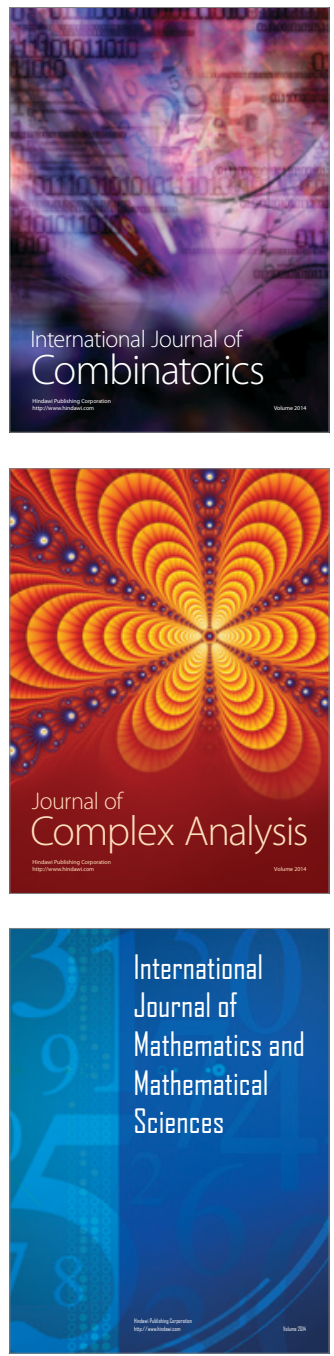
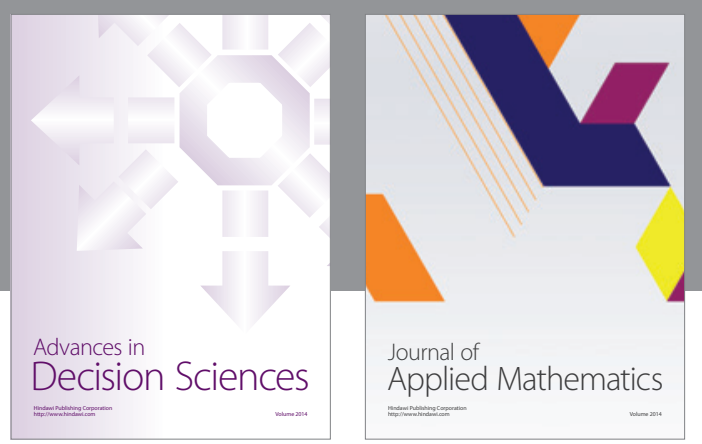

Algebra

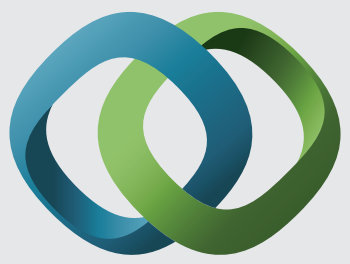

\section{Hindawi}

Submit your manuscripts at

http://www.hindawi.com
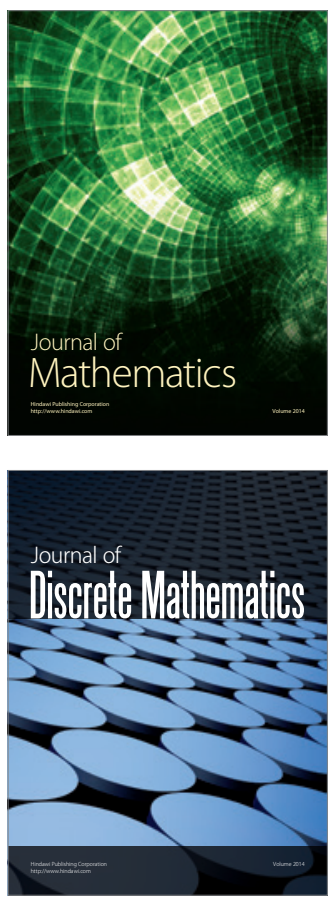

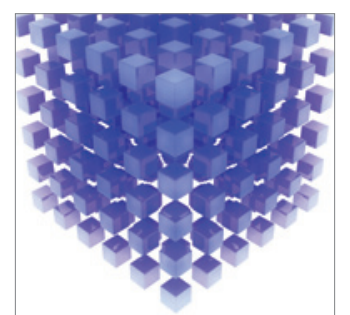

Mathematical Problems in Engineering
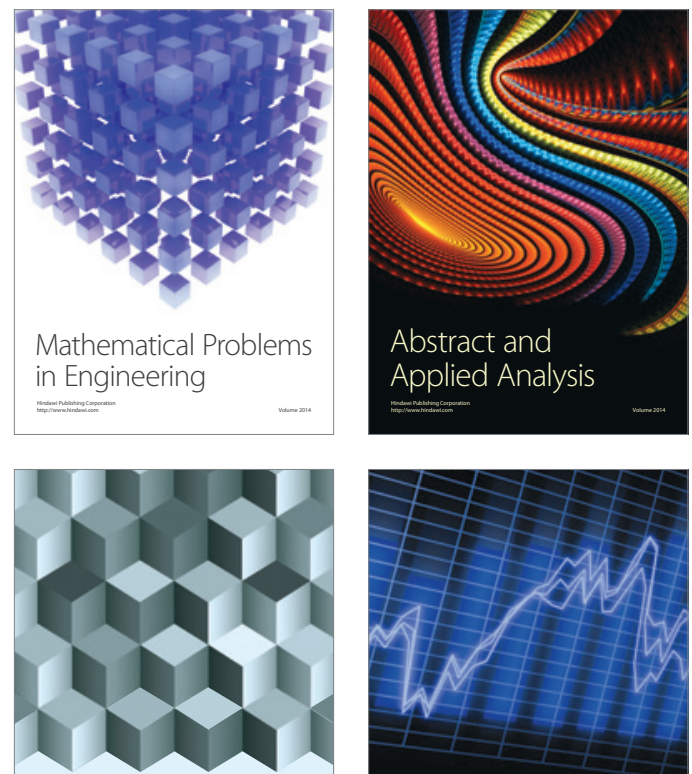

Journal of

Function Spaces

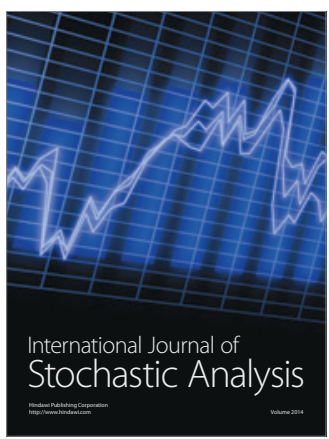

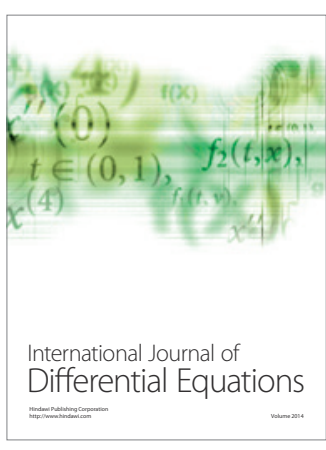
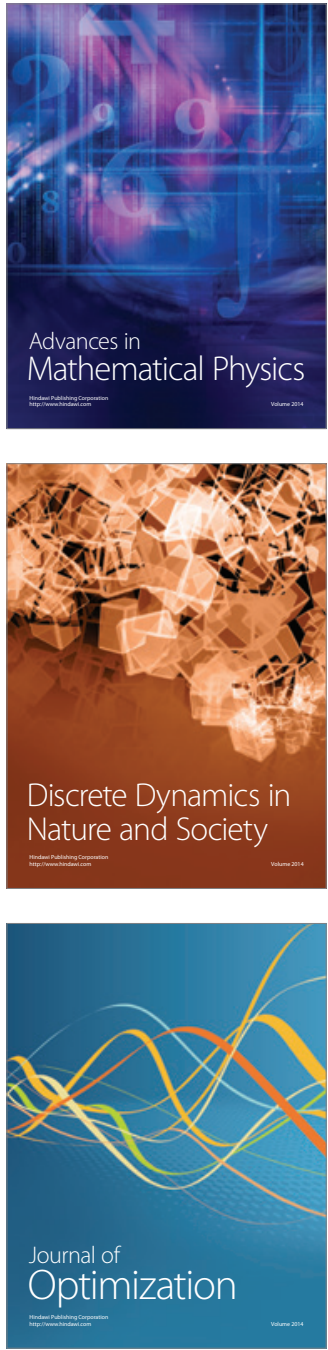